Military Technical College Kobry El-Kobbah, Cairo, Egypt

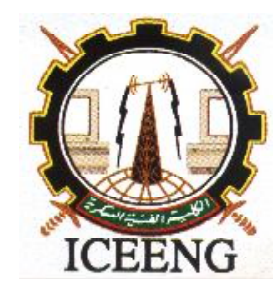

$9^{\text {th }}$ International Conference on Electrical Engineering ICEENG 2014

\title{
Flashover Performance of contaminated HV Outdoor Insulators in Egypt
}

By

\author{
M. M. Abouelsaad* \\ M. A. Abouelatta * \\ B. A. Arafa \\ M. E. Ebrahim
}

\section{$\underline{\text { Abstract: }}$}

Contamination on outdoor insulators enhances the chances of flashover and can lead to transmission line outages and reduced system reliability. In practice, various contaminant types, usually classified as soluble and insoluble, settle on outdoor insulators and increase the possibility of flashover. The paper assesses the flashover performance of such insulators in Egypt; in view of the nature and composition of contaminants accumulated on their surfaces. To quantify these contaminants, numerous samples are gathered from different regions of the country and subjected to a comprehensive chemical analysis at the scale analysis laboratory of the Egyptian Electricity Transmission Company. Measurements of equivalent salt deposition density (ESDD) surface conductivity (SC), maximum leakage current (MLC) and flashover voltage (FOV) were conducted and correlated to the contamination constituents. The study utilized IEC standard cap-pin suspension insulators which were hanged in regions of different pollution levels and nature. Regression models for predicting the flashover voltage as related to ESDD and MLC as related to surface conductivity were developed. The models are validated through statistical analysis as well as comparison with measured data. The results from this work are useful for utilities to take adequate measures to improve the efficiency of their transmission systems through scheduling of their insulator maintenance strategy and selecting the appropriate insulator design for different system voltages and regions.

\section{Keywords:}

Outdoor Insulators, ESSD, Contaminations, Surface Conductivity

* Shoubra Faculty of Engineering, Egypt 


\section{Introduction:}

High voltage outdoor insulators are, usually, subject to different pollution sources; namely, industrial, desert and marine pollution, which may cause insulator's flashover and discontinuity of supply; sometimes on a large scale $[1,2]$. The possibility of flashover in contaminated environments depends on the type of pollution and the time that the insulator is subjected to such pollution [3]. A contamination layer is formed on the insulator's surface and may allow leaking currents that will facilitate the conditions of flashover. The constitution of this pollution differs from one site to another. The performance of insulator under contaminated conditions is the underlying factor that determines insulation design for outdoor application [4].

Under dry conditions, contaminated surfaces do not conduct so contamination is of little concern. Under environmental conditions of light rain, fog or dew, surface contamination dissolves [5,6]. This forms a conducting layer on the surface of the insulator which facilitates a leakage current. High current density near the electrodes heats and dries the pollution layer. An arc is initiated; if the voltage stress across the insulator's dry band exceeds its withstand capability. Extension of the arc across the insulator ultimately results in flashover. The contamination severity determines the frequency and intensity of arcing and, thus, the probability of flashover [6].

Insulators located near coastal regions are typically contaminated by soluble contaminants, especially salt (or sodium chloride) while insulators located near cement or paper industries are typically contaminated by non-soluble contaminants such as calcium chloride, carbon and cement dust. Irrespective of the type of contaminant, flashover can occur as long as the salts in the contaminant are soluble enough to form a conducting layer on the insulator's surface [5,6]. The flashover characteristics are influenced by the chemical composition of contaminants [7].

During the last few years, large industrial developments in various regions of Egypt have contributed to producing more contaminations that could, largely, affect the power lines' insulators performance. Heavy industry factories (cement, quarries, steel \& iron, etc.) have been established in desert and coastal regions resulting in mixtures of different pollution types [8]. It is, thus, necessary to evaluate the flashover performance of these insulators in correlation of the prevailing contamination nature and composition in Egypt.

In the present study, measurements of flashover voltage (FOV), maximum leakage current (MLC) and surface conductivity (SC) of contaminated cap-pin suspension insulators, gathered from several regions with different types and levels of pollutions, 
were conducted at the Pyramids High Voltage Research Center (PHVRC), Cairo, Egypt. In addition, typical chemical analysis of contamination constituents, obtained by x-ray diffraction analysis in the Scale Analysis Laboratory of the Egyptian Electricity Transmission Company, is carried out and correlated to the above measurements. The performance of insulators is assessed through measurements of; ESDD and chemical analysis to examine the nature of the contamination materials. Regression models for predicting the FOV as related to ESDD and MLC as related to surface conductivity were developed. The models are validated through statistical analysis as well as comparison with measured data. The results are utilized to assess the insulation performance in different environmental situations and to modify the design if necessar.

\section{Contamination Severity \& Measurements:}

In order to establish the effect of variation of ESDD on the flashover voltage FOV, a series of experiments were performed on naturally contaminated samples of insulators. IEC standard cap-pin suspension insulators, shown in Figure (1), were hanged along $66 \mathrm{kV}$ and $220 \mathrm{kV}$, for 5 months during the winter season, in regions with various types and intensities of pollution. The pollution characteristics of these regions are as follows:

1. Region 1: A mixture of costal / industrial contamination exist (66kV TL inside a cement factory located $2 \mathrm{~km}$ away from the Mediterranean).

2. Region 2: Coastal (220kV TL inside a steam power plant operated with fuel oil and natural gas). The region is exposed to heavy rain and salted dew effects especially in the winter season.

3. Region 3 Coastal and Industrial (220kV TL inside a steel factory). The region is close to Salinas region. Both marine and industrial pollution exist.

4. Region 4 Desert and Industrial ( $66 \mathrm{kV}$ TL beside a cement factory). The area is exposed to heavy sandstorms.

5. Region 5: Desert and Quarries (66kV TL beside Quarries and cement factory).

6. Region 6: Industrial (66kV TL beside several Bricks Kiln with high chimneys which produce carbon leftovers)

\section{Measurement of Surface Conductivity (SC) \& ESDD}

The performance of insulators performance depends on the contamination severity on the surface of insulators; usually given in terms of ESDD. The standard procedure for measurement of ESDD has been standardized in the IEC-60507. ESDD is measured by dissolving the contaminants on the surface of the insulators, in deionized water and then measuring the conductivity of the water. The ESDD is calculated using the following formula [9] 


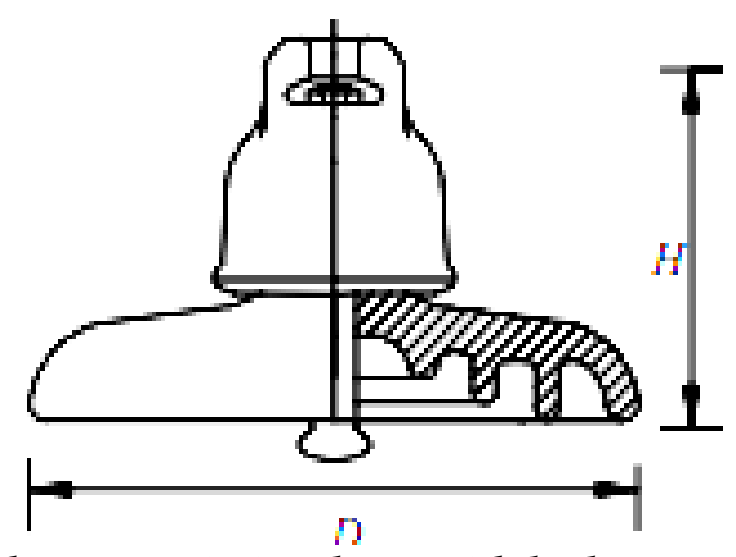

Figure (1): Profile of the cap - pin insulator with leakage path $215 \mathrm{~mm}, D=280 \mathrm{~mm}$, and $H=146 \mathrm{~mm}$

$\sigma_{20}=\sigma_{\theta}(1-b(\theta-20))$

$\sigma_{\theta}$ - The measured conductivity in $\mathrm{S} / \mathrm{m}$.

b - 0.01998 (constant)

$\theta$ - Temperature of the solution $\left({ }^{\circ} \mathrm{C}\right)$

$\mathrm{ESDD}=\left(5.7 * \sigma_{20}\right)^{1.03} * \mathrm{VO} / \mathrm{AR}$

VO- Volume of dissolvent (distilled water) in $\mathrm{m}^{3}$

$\mathrm{AR}$ - Area of the insulator surface in $\mathrm{m}^{2}$

This method is observed to be good for measuring ESDD for porcelain and glass insulators [6].

Measurements of flashover voltage (FOV), maximum leakage current (MLC), ESDD and surface conductivity (SC) were conducted according to standard procedures [9-11] at the Pyramids High Voltage Research Center (PHVRC) of Egypt. Results of the average values are reported in Table I. As expected, higher values of ESSD result in an increase in Maximum leakage current and Surface conductivity but lower flashover voltage.

Table (1): Experimental results for ESDD, FOV, MLC and SC

\begin{tabular}{|c|c|c|c|c|c|c|}
\hline ESDD $\left(\mathbf{m g} / \mathbf{c m}^{\mathbf{2}}\right)$ & 0.130 & 0.208 & 0.22 & 0.53 & 1.34 & 1.37 \\
\hline MLC $(\mathbf{m A})$ & 2.733 & 4.133 & 4.26 & 14.66 & 29 & 48.26 \\
\hline FOV $(\mathbf{k V})$ & 29.163 & 25.64 & 22.88 & 15.64 & 7.73 & 4.909 \\
\hline SC $(\boldsymbol{\mu S})$ & 6.653 & 9.47 & 9.86 & 37.35 & 80.42 & 133.1 \\
\hline
\end{tabular}




\section{Evaluation and Modeling of Contamination Flashover Performance}

From table I, it is noticed that the tests have been made for levels of ESDD ranging from 0.13 and 1.37. It is possible to develop a regression model to predict, with a high prediction interval of 95\%, the FOV for other levels of ESDD, especially

lower levels, where experimental data may not be available. In order to consider the model valid, it is necessary that the fitted regression model can be validated at some other points where it has been extrapolated.

To justify modeling the data, the statistical correlation between the variables ESDD and FOV is established. The correlation- coefficient matrix is calculated to be;

$$
\begin{array}{rr}
1.0000 & -0.9749 \\
-0.9749 & 1.0000
\end{array}
$$

The diagonal elements are equal to 1; representing the perfect correlation of each variable with itself. There is a strong statistical correlation between the variables ESDD and FOV with the off- diagonal elements being very close to 1 .

The regression equation is assumed to have the following form:

$\mathrm{FOV}=\mathrm{a}_{0}+\mathrm{a}_{1} * \ln (\mathrm{ESDD})$

The range of ESDD used for developing the regression model is 0.208 to 1.37. Employing linear regression model, using MATLAB programming [12], the values of $a_{0}, a_{1}$ are found to be 9.317 and -9.831 respectively.

Therefore, the model of the data is given by

$\mathrm{FOV}=9.317+9.831 * \ln (\mathrm{ESDD})$

Figure 2 shows the FOV fit for the experimental results reported in Table I. Also shown are the 95\% upper and lower limits and the data used in the regression model and the data to be predicted using the developed regression model.

The statistical characteristics of the developed regression model are presented in Fig.3; as certain assumptions must be satisfied in order for the model to be considered valid. It can be seen from the normal probability plot of residuals that the 
residuals lie approximately in a straight line; meaning that there is no considerable deviation from normality. The first assumption of normality of errors is thus satisfied. Typically, the points should be randomly scattered in order to satisfy the assumption of constant variance. It is clear that the plot between the residuals and predicted values does not indicate any specific shape. The check that errors should be uncorrelated is realized in the plot between residuals and ESDD as there is no specific trend discernable from the plot. Independence of errors is thus realized. As all the assumptions were checked and satisfied; hence, the developed model is considered valid to represent the data.

In order to validate the model in the region of extrapolation a data point for ESDD of 0.13 was considered. Predicted values for new observations at $95 \%$ are:

Prediction Interval $=($ lower limit $28.04 \mathrm{kV}$, upper limit $31.84 \mathrm{kV})$ and From Experiment $=29.16 \mathrm{kV}$.

Predicted value $=29.25 \mathrm{kV}$

The experimentally obtained value is observed to be within the range of $95 \%$ prediction limits and thus the model is validated.

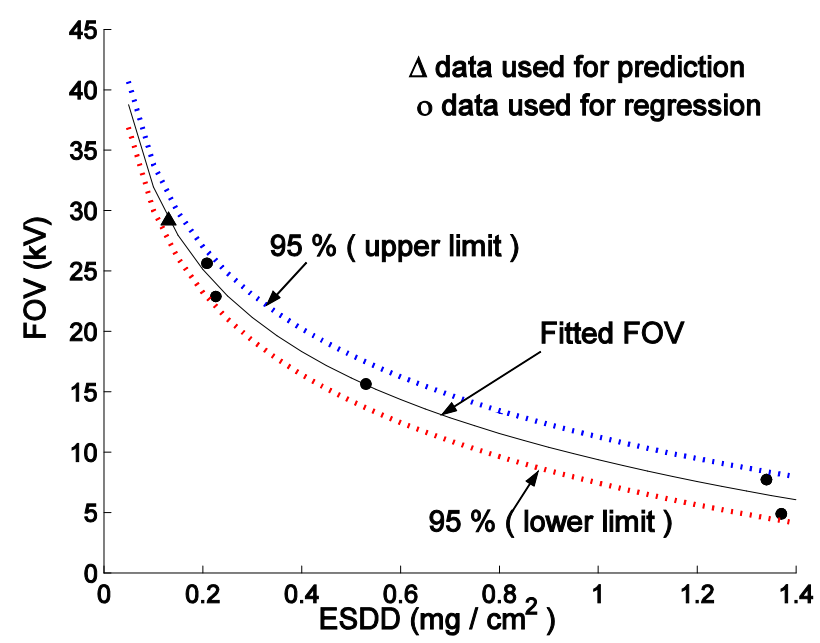

Figure (2): FOV prediction curve at 95\% Prediction interval for the cap - pin insulator 

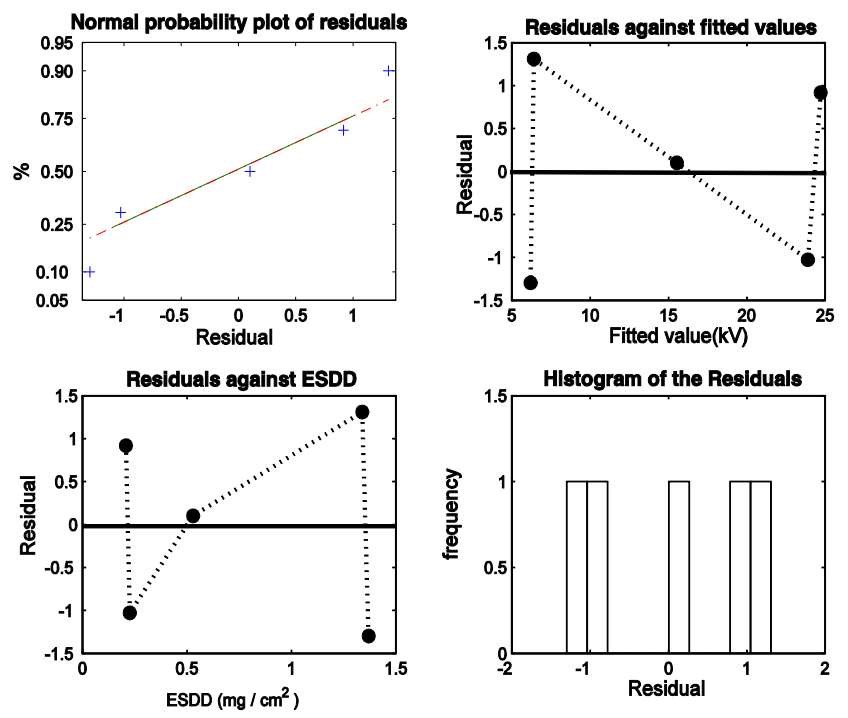

Figure (3): Check of the statistical assumptions for the developed model

\section{Measurement \& Modeling of Maximum Leakage Current (MLC)}

Measurement of maximum leakage current is an effective indicator for evaluating the performance of contaminated insulators on transmission lines. In case of flashover, the maximum leakage current value is defined as the peak current value in the half cycle before flashover [10]. The experimental results obtained against the measured surface conductivity (SC) are reported in Table I and plotted in Fig.4.

Similar to the procedures for the flashover performance, it is possible to develop a regression model to predict, with a high prediction interval of 95\%, the MLC for other levels of SC, where experimental data may not be available.

The correlation- coefficient matrix is calculated to be;

$$
\begin{array}{cc}
1.0000 & 0.9998 \\
0.9998 & 1.0000
\end{array}
$$

There is a strong statistical correlation between the variables SC and MLC with the offdiagonal elements (0.9998) being very close to 1.

The regression equation is assumed to have the following form:

$\mathrm{MC}=\mathrm{b}_{0}+\mathrm{b}_{1} *(\mathrm{SC})$ 
The range of SC used for developing the regression model is 9.47 to 133.1. Employing linear regression model, the values of $b_{0}, b_{1}$ are found to be 0.355 and 0.897 respectively.

$$
\mathrm{MC}=0.355+0.897 *(\mathrm{SC})
$$

Figure 4 shows the MLC fit for the experimental results reported in Table I. Also shown are the 95\% upper and lower limits and the data used in the regression model and the data to be predicted using the developed regression model. All the assumptions, discussed earlier, were checked and satisfied; hence, the developed model is considered valid to represent the data.

In order to validate the model in the region of extrapolation a data point for SC of 6.653 was considered. Predicted values for new observations at $95 \%$ are:

Prediction Interval $=($ lower limit $2.34 \mathrm{~mA}$, upper limit $3.84 \mathrm{~mA})$ and From Experiment $=2.733 \mathrm{~mA}$.

Predicted value $=3.24 \mathrm{~mA}$

The experimentally obtained value is observed to be within the range of 95\% prediction limits and thus the model is validated.

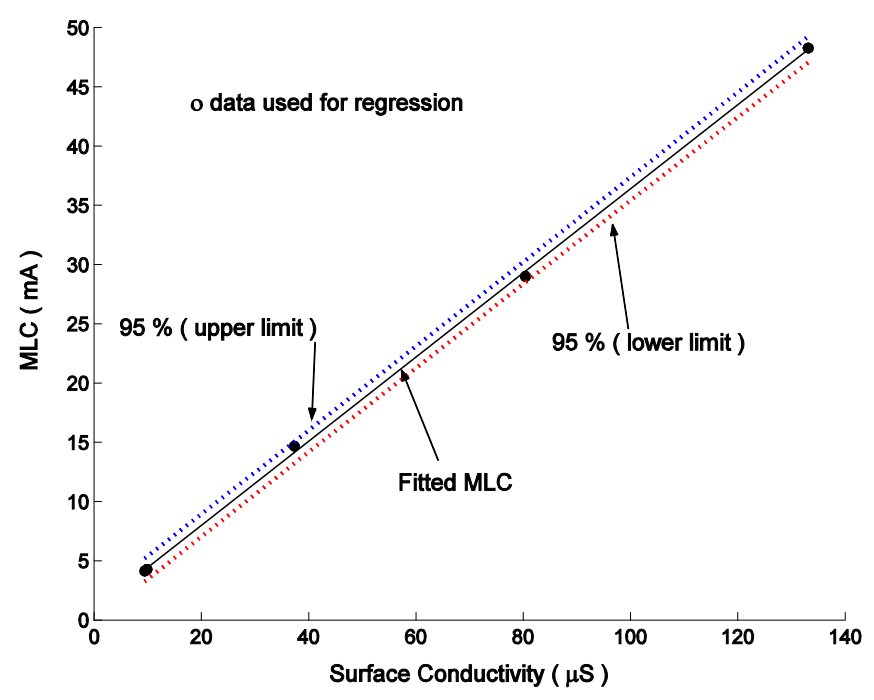

Figure (4): Maximum Leakage Current against Surface Conductivity

\section{Chemical Analysis Of The Contaminants Accumulated On Insulators:}

Egypt is a country with a great variety of climates and topographical conditions, so high 
voltage insulators are exposed to various chemical contaminations which affect the flashover performance of power lines insulators. The contaminants deposited upon the specimens gathered from the considered regions were subjected to chemical analysis in the scale analysis laboratory, Egyptian Electricity Transmission Company. Table II and Table III record a summary of the major chemical elements, obtained by x-ray diffraction analysis, contained in the examined samples. Also, Table IV and Figure (5) report $\mathrm{pH}$ measurements and the percentage of dissolved and non- dissolved materials respectively. This chemical analysis should be of help in evaluating the resultant ESDD and electrical conductivity measurements. Inspection of the chemical analysis data reveals the following observations;

- The silica percentage $\left(\mathrm{SiO}_{2}\right)$ is rather high in Regions 4,5 and 6 compared to other regions. This may be due to the closeness of these regions to desert areas and also due to the presence of non-dissolved carbon magma upon the insulator surface (Region 6).

- The ferric oxide percentage $\left(\mathrm{Fe}_{2} \mathrm{O}_{3}\right)$ in Region 3 has the largest value which may be due to its vicinity to iron factory. Regions 5 and 6 have relatively high values of this element due the presence of several Bricks Kiln in these regions (cement powder usually include iron element).

- The highest percentage of dissolved material is the one which has the highest Chlorine percentage $(\mathrm{Cl})$. This is expressed in Region 6. This chloride salts dissolve in water and when the dissolving rate increases, the electrical conductivity increases.

- Samples with low $\mathrm{pH}$ value have the highest $\mathrm{Cl}$ percentage which may lead to higher electrical conductivity as the chloride salts are ionized during dissolving. So, Region 6 has the lowest $\mathrm{pH}$ value. But out of this pattern, Region 5 has a very high $\mathrm{pH}$ value because the calcium percentage $(\mathrm{CaO})$ in the Quarries is rather high which converts into calcium hydroxide during wetness (a strong alkaline material). Also, although Region 2 has large $\mathrm{CaO}$ percentage, the $\mathrm{pH}$ value is not very high like Region 6 due to the presence of fuel oil and natural gas.

Increasing nitrogen percentage in the specimens' leads to acids formation which reduces the $\mathrm{pH}$ values.

The above analysis indicates the strong dependence of the contamination constituents on the prevailing environment in the neighborhood of power lines. This; in turn, influence the insulators' performance; mainly the surface conductivity, the flashover voltage and the maximum leakage current. As an example, insulators in the region with highest 
dissolvable materials (highest chloride from the chemical analysis) will have the highest leakage current and lowest flashover voltage as given on Figs. 2, 4. This is due to the increased surface conductivity in this case. The present study is useful for utilities in order to evaluate and assess the environment impact of pollution on the insulators' performance. While in some cases it may be advisable to require factories to use suitable filters to reduce emissions and contaminants emanating from them, it may be necessary in other cases to avoid insulators' flashover through appropriate selection and/or design of the insulators (shape, length and number). In addition, adequate scheduled maintenance plan and strategy can be developed to achieve the required insulators' performance.

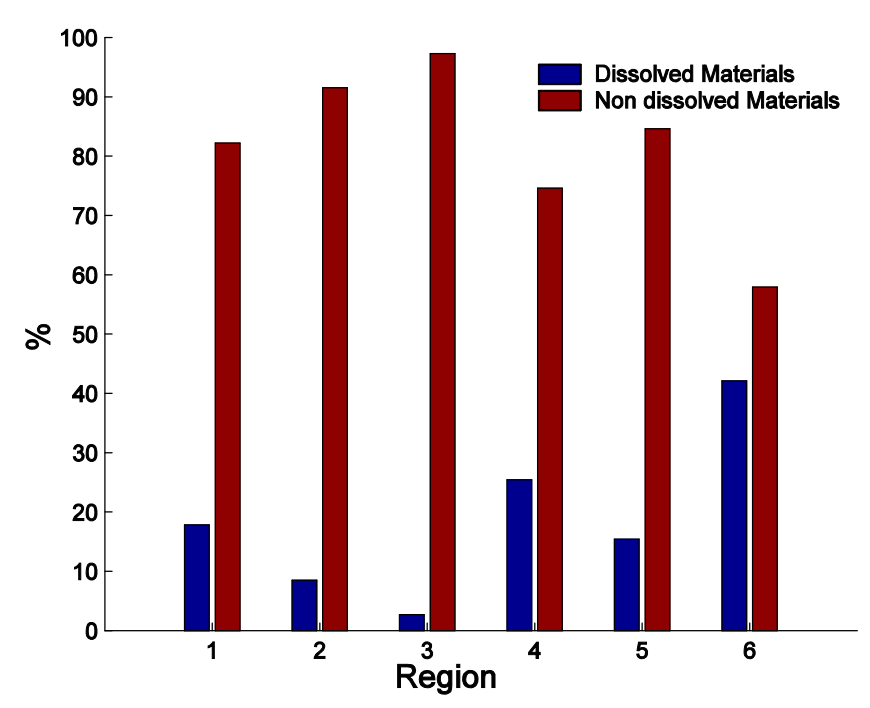

Figure (5): Percentage of Dissolved and Non-dissolved materials for the different regions

Table (2): Percentage chemical contaminants upon insulator

\begin{tabular}{|c|c|c|c|c|c|}
\hline Region & $\mathbf{\% ~} \mathbf{S i O}_{\mathbf{2}}$ & $\mathbf{\% F e}_{\mathbf{2}} \mathbf{O}_{\mathbf{3}}$ & $\mathbf{\%} \mathbf{C a O}$ & $\mathbf{\%} \mathbf{M g O}$ & \% Cl \\
\hline $\mathbf{1}$ & 8.1 & 0.56 & 34.2 & 15 & 3.1 \\
\hline $\mathbf{2}$ & 3.8 & 0.25 & 25.8 & 18.7 & 0.29 \\
\hline $\mathbf{3}$ & 10.6 & 11.1 & 23.9 & 17.5 & 0.22 \\
\hline $\mathbf{4}$ & 21.2 & 4 & 26.1 & 3 & 4.9 \\
\hline $\mathbf{5}$ & 32.5 & 4.52 & 27.5 & 7.9 & 1.7 \\
\hline $\mathbf{6}$ & 33.06 & 5.1 & 9.2 & 7.8 & 7.3 \\
\hline
\end{tabular}


Table (3): Chemical contaminants upon insulator

\begin{tabular}{|c||c|c|c|}
\hline Region & $\mathbf{N H}_{\mathbf{3}}(\mathbf{M g} / \mathbf{k g})$ & $\left.\mathbf{N O}_{\mathbf{3}} \mathbf{( M g} / \mathbf{k g}\right)$ & $\begin{array}{c}\text { Oil and Greases } \\
(\mathbf{M g} / \mathbf{k g})\end{array}$ \\
\hline $\mathbf{1}$ & 210 & 6.9 & 3000 \\
\hline $\mathbf{2}$ & 268 & 309 & 2500 \\
\hline $\mathbf{3}$ & 640 & 25 & 2000 \\
\hline $\mathbf{4}$ & 320 & 8850 & 1400 \\
\hline $\mathbf{5}$ & 102 & 152 & 2400 \\
\hline $\mathbf{6}$ & 2240 & 7000 & 1500 \\
\hline
\end{tabular}

Table (4): $p H$ measurements for the different regions

\begin{tabular}{|c|c|c|c|c|c|c|}
\hline Region & $\mathbf{1}$ & $\mathbf{2}$ & $\mathbf{3}$ & $\mathbf{4}$ & $\mathbf{5}$ & $\mathbf{6}$ \\
\hline $\mathbf{p H}$ & 8.6 & 8.32 & 8.64 & 7.3 & 10.36 & 6.35 \\
\hline
\end{tabular}

\section{Conclusions:}

Measurements of equivalent salt deposition density (ESDD), flashover voltage (FOV), maximum leakage current (MLC) and surface conductivity (SC) of contaminated cappin suspension insulators, gathered from several regions with different types and levels of pollutions, were conducted. In addition, typical chemical analysis of contamination constituents is carried out and correlated to the above measurements. Regression models for predicting the FOV as related to ESDD and MLC as related to SC were developed. The models are validated through statistical analysis as well as comparison with measured data. The results from this work are useful for utilities to take adequate measures to improve the efficiency of their transmission systems through scheduling of their insulator maintenance strategy and selecting the appropriate insulator design for different system voltages and regions.

\section{References:}

[1] E. Feilat, A. Al-Maqrashi, "ESDD- and DDDG- based assessment of insulator pollution levels in Oman", Proc. of the $6^{\text {th }}$ IEEE GCC Conference \& Exhibition 2011, UAE, pp. 593-596, February 2011.

[2] P. Lambeth, "Effect of pollution on high-voltage outdoor insulators", IEE Reviews, vol. 118, No. 9, pp. 1107- 1131, 1971. 
[3] S. Nabavi, A. Gholami, A. Kazemi, M. Masoum, "Evaluation of leakage current measurement for site pollution severity assessment", Leonardo Journal of Practices and Technologies, vol. 10, pp. 39-54, 2004.

[4] Waluyo, P. Pakpahan, Suwarno, M. Djauhari "Comparative Study on Properties of Coastal Polluted Porcelain and Epoxy Resin Outdoor Insulators", International Journal of Computer and Information Engineering, Vo. 2, pp. 171-179, 2008.

[5] Venkataraman , R. Gorur ," Prediction of Flashover Voltage of Non-ceramic Insulators Under Contaminated Conditions", IEEE Transactions on Dielectrics and Electrical Insulation Vol. 13, No. 4, pp 862- 869, 2006.

[6] R. Gorur, R. Olsen ," Prediction of Flashover Voltage of Insulators Using Low Voltage Surface Resistance Measurement", Power Systems Engineering Research Center Publication 06-42, 2006.

[7] M. Slama, A. Beroual, H. Hadi, " Influence of Pollution Constituents on DC Flashover of High Voltage Insulators", IEEE Transactions on Dielectrics and Electrical Insulation Vol. 20, No. 2, pp 401- 408, 2013.

[8] M..Abouelsaad, M.A.Abouelatta, B.Arafa, M. Ibrahim, "Assessment of Pollution on Outdoor High Voltage Insulators In Egypt", $18^{\text {th }}$ International Symposium of High Voltage Engineering ISH 2013, Korea, 28-30 August, 2013.

[9] IEC 60507, $2^{\text {nd }}$ Ed., "Artificial Pollution Tests on High - Voltage Insulators to Be Used on AC Systems", April 1991.

[10] I. Ramirez, R. Hernandez, G. Montoya: “ Measurement of leakage current for monitoring the performance of outdoor insulators in polluted environments", IEEE Electrical insulation magazine, Vol.28, pp. 29-34, 2012.

[11] Y. Xia, X. Jiang, C. Sun, B. Dong: "A method to estimate leakage current of polluted insulators", Przeglad Elektrotechniczny, Vol.88, pp. 161-164, 2012.

[12] D. Hanselman, B. Littlefield, "Mastering MATLAB 6 - A Comprehensive Tutorial and Reference", Prentice Hall, 2000. 